\title{
Human-Driven FOL Explanations of Deep Learning
}

\author{
Gabriele Ciravegna ${ }^{1,2}$, Francesco Giannini ${ }^{2}$, Marco Gori $^{2,3}$ \\ Marco Maggini ${ }^{2}$ and Stefano Melacci $^{2 *}$ \\ ${ }^{1}$ Department of Information Engineering, University of Florence, Florence, Italy \\ ${ }^{2}$ SAILab, Department of Information Engineering and Mathematics, University of Siena, Siena, Italy \\ ${ }^{3}$ Maasai, Universitè Côte d'Azur, Nice, France \\ gabriele.ciravegna@unifi.it,\{fgiannini,mela,maggini,marco\}@diism.unisi.it
}

\begin{abstract}
Deep neural networks are usually considered blackboxes due to their complex internal architecture, that cannot straightforwardly provide humanunderstandable explanations on how they behave. Indeed, Deep Learning is still viewed with skepticism in those real-world domains in which incorrect predictions may produce critical effects. This is one of the reasons why in the last few years Explainable Artificial Intelligence (XAI) techniques have gained a lot of attention in the scientific community. In this paper, we focus on the case of multilabel classification, proposing a neural network that learns the relationships among the predictors associated to each class, yielding First-Order Logic (FOL)-based descriptions. Both the explanationrelated network and the classification-related network are jointly learned, thus implicitly introducing a latent dependency between the development of the explanation mechanism and the development of the classifiers. Our model can integrate human-driven preferences that guide the learningto-explain process, and it is presented in a unified framework. Different typologies of explanations are evaluated in distinct experiments, showing that the proposed approach discovers new knowledge and can improve the classifier performance.
\end{abstract}

\section{Introduction}

In the last few years the scientific community devoted a lot of effort to the proposal of approaches that yield explanations to the decisions of machine learning-based systems [Bibal and Frénay, 2016; Doshi-Velez and Kim, 2017; Došilović et al., 2018; Guidotti et al., 2018; Teso and Kersting, 2019]. In particular, several Explainable Artificial Intelligence (XAI) [Gunning, 2017] techniques have been developed, with different properties and output formats. They generally rely on existing interpretable models, such as decision trees, rules, linear models [Freitas, 2014; Huysmans et al., 2011], that are considered easily understandable by

\footnotetext{
${ }^{*}$ Contact Author
}

humans. On the other hand, in order to provide an explanation for black-box predictors, such as (deep) neural networks and support vector machines, a new interpretable model that is as faithful as possible to the original predictor is considered, sometimes acting on localized regions of the space [Guidotti et al., 2018]. Then, the explanation problem consists in finding the best interpretable model approximating the black-box predictor. In the context of the XAI literature, there is no clear agreement on what an explanation should be, nor on what are the suitable methodologies to quantitatively evaluate its quality [Carvalho et al., 2019; Molnar, 2019]. There is also a strong dependence on the target of the explanation, e.g., a common user, an expert, or an artificial intelligence researcher.

In this paper, we consider multi-label classification, where each input example belongs to one of more classes, and on First-Order Logic (FOL)-based explanations of the behaviour of the classifier. We focus on neural network-based systems, that implicitly learn from supervisions the relationships among the considered classes. We propose to introduce another neural network that operates in the output space of the classifier, also referred to as concept space, further projecting the data onto the so-called rule space, where each coordinate represents the activation of a rule/explanation that, afterwards, is described by FOL. In particular, we propose to progressively prune the connections of the newly introduced network and interpret each of its neurons as a learnable boolean function (an idea related to several methods [Fu, 1991; Towell and Shavlik, 1993; Tsukimoto, 2000; Sato and Tsukimoto, 2001; Zilke et al., 2016]), ending up in a FOL formula for each coordinate of the rule space.

The concepts-to-rules projection can be learned using different criteria, that bias the type of rules discovered by the system. We propose a general unsupervised criterion based on information principles, following [Melacci and Gori, 2012]. However, humans usually have expectations on the kind of explanations they might get. For example, suppose we are training a network to classify digits and also to predict whether they are even numbers. If we do not know what being even means, we might be particularly interested in knowing the relationships between the class even and the other classes (i.e., that even numbers are 0 or 2 or 4 or 6 or 8 ). It could not be so useful to discover that 0 is not 2 , even if it is still a valid explanation in the considered multi-label problem. Mo- 
tivated by this consideration, we propose a generic framework that can discover both unbiased and user-biased explanations.

A key feature of the proposed framework is that learning the classifier and the explanation-related network takes place in a joint process, differently from what could be done, for example, by classic data mining tools [Liu, 2007; Witten and Frank, 2005]. This implicitly introduces a latent dependency between the development of the explanation mechanism and the one of the classifiers. When cast into the semi-supervised learning setting, we show that linking the two networks can lead to better quality classifiers, bridging the predictions on the unsupervised portion of the training data by means of the explanation net, that acts as a special regularizer.

The paper is organized as follows. Section 2 introduces the use cases covered in this paper, while the proposed model is described in Section 3. Experiments are collected in Section 4 and Section 5 concludes the paper.

\section{Scenarios}

We consider a multi-label classification problem, in which a multi-output classifier is learned from data. Each output unit is associated to a function in $[0,1]$ that predicts how strongly an input example belongs to the considered class. We will also interchangeably refer to these functions as task functions (in a more general perspective where each function is related to a different task), or predicates (if we interpret each output score as the truth value of a logic predicate).

We also consider a set of explanations, that express knowledge on the relationships among the task functions, and that are the outcome of the proposed approach. Such knowledge is not known in advance, and it represents a way to explain what the classifier implicitly learned about the task functions. In order to guide the process of building the explanations, the user can specify one or more preferences. In particular, the user can decide if the explanations have to describe local relationships that only hold in sub-portions of the concept space or global rules that hold everywhere, or even if they must focus on a user-selected task function (as in the example of Section 1). In what follows we report an overview of the specific use cases explored in this paper.

Local Explanations. In this scenario, the explanations are automatically produced without making any assumptions on which task functions to consider. In order to provide a valid criterion to develop explanations, we enforce them to only hold in sub-portions of the concept space and, overall, to cover the whole dataset. The user can provide an example to the trained network and get back the explanation associated to it, that may highlight partial co-occurrences of the task functions. For instance, the system might discover that "eyes or sunglasses" is a valid rule for some pictures (the ones with faces) but not for others (the ones without faces).

Global Explanations. Local explanations may provide very specific knowledge concerning only small portions of data. In order to describe more general properties that hold on the whole dataset, we may be interested in global explanations. Global explanations may catch general relations among task functions that are valid for all the points of the considered dataset, such as mutual exclusion of two classes or hierarchical relations.

Class-driven Explanations. The user may require explanations about the behaviour of specific task functions. He could also specify if he is looking for necessary conditions $(I F \rightarrow)$ or necessary and sufficient explanations $(I F F \leftrightarrow)$. For instance, focusing on the driving class man, we may discover that a certain pattern is classified as "man only if it is also classified as containing hand, body, head", and so on. In the example of Section 1, even was the class driving a necessary and sufficient explanation. The rules of this scenario are completely tailored around the user-selected target classes.

Combined Explanations. All the scenarios described so far may be arbitrarily combined in case the user is simultaneously interested in multiple explanations according to different criteria. In particular, some explanations might have to specify the behaviour of some task functions, while the remaining ones might have to be automatically acquired in order to describe global or local interactions.

\section{Model}

We consider data belonging to the perceptual space $X \subseteq \mathbb{R}^{d}$, and $n$ labels/classes, each of them associated to a task function $f_{i}, i=1, \ldots, n$, that corresponds to an output unit of a neural network. For any $x \in X, f_{i}(x) \in[0,1]$ expresses the membership degree of the example $x$ to the $i$-th class. We indicate with $f(x)$ the function that returns the $n$-dimensional vector with the outputs of all the task functions. Such vector belongs to the so-called concept space.

Let us consider another set of functions implemented by neural networks, indicated with $\psi_{j}, j=1, \ldots, m$, whose input domain is the concept space while their output domain is the rule space. Each $\psi_{j}(f(x))$ expresses the validity of a certain explanation with respect to the output of the task functions on the data sample $x \in X$. In addition, we assume $\psi_{j}(f(x)) \in[0,1]$ in order to relate the value of $\psi_{j}$ to the truth-degree of a certain FOL formula.

Different criteria are needed to learn the parameters of the functions $\psi_{j}$ in order to implement the scenarios of Section 2, as we will describe in Section 3.1. Once the explaining functions are learnt, we will consider their approximation as boolean functions, and they will be given a description in terms of FOL, as we will discuss in Section 3.2. Throughout the paper, the notation $\hat{\psi}_{j}$ denotes both the approximating boolean function and its associated logical formula. Finally, $X_{j}$ denotes the subset of the input space where the $j$-th explanation holds true, also named its support, i.e., $X_{j}=\left\{x \in X: \hat{\psi}_{j}(f(x))=1\right\}$. When no subscript is specified, $f$ and $\psi$ indicate the collection of all the $f_{i}$ 's and $\psi_{j}$ 's, respectively.

\subsection{Learning Criteria}

We consider a semi-supervised setting in which only a portion of the data in $X$ is labeled [Melacci and Belkin, 2011]. This is a natural setting of several real-world applications, since getting labeled data is usually costly, and it also allows us 
to better emphasize the proprieties of the explanation learning mechanisms, that can exploit both labeled and unlabeled training data with no distinctions.

The classic cross-entropy loss is used to enforce the task functions $f_{i}$ 's to fit the available supervisions, paired with a regularization criterion to favour smooth solutions (weight decay). In order to implement the scenarios of Section 2, we need to augment the training loss with further criteria (penalty terms) that involve the explaining functions $\psi_{j}$ 's, for all $x$ 's, being them labeled or not, and described in what follows. ${ }^{1}$

Mutual Information-based Criterion. The maximization of the Mutual Information (MI) between the concept and rule spaces can be enforced in order to implement the principles behind the Local Explanations scenario, and it could also be used as a basic block to implement the Global Explanations scenario (Section 2). In the latter case, further operations are needed, and they will be described in Section 3.2. Maximizing the transfer of information from the $n$ task functions to the $m$ explaining functions is a fully unsupervised process that leads to configurations of the $\psi_{j}$ 's functions such that, for each $x \in X$, only one of them is active (close to 1 ) while all the others are close to zero (see [Melacci and Gori, 2012]). In order to define the MI index, we introduce the probability distribution $P_{\Psi=j \mid Y=f(x)}(\psi, f(x))$, for all $j$, as the probability of $\psi_{j}$ to be active in $f(x)$. Following the classic notation of discrete MI, $\Psi$ is a discrete random variable associated to the set of explaining functions while $Y$ is the variable related to the data in the concept space. ${ }^{2}$ The penalty term to minimize is minus the MI index, that is

$$
L_{M I}(\psi, f, X)=-H_{\Psi}(\psi, f, X)+H_{\Psi \mid Y}(\psi, f, X),
$$

where $H_{\Psi}$ and $H_{\Psi \mid Y}$ denote the entropy and conditional entropy functions (respectively) associated to the aforementioned probability distribution and measured over the whole $X$. An outcome of the maximization of the MI index is that the supports of the explaining functions will tend to partition the input space $X$, i.e., $X=\bigcup_{j=1}^{m} X_{j}$ and $X_{j} \cap X_{k}=\emptyset$, for $j \neq k$ (see [Melacci and Gori, 2012; Betti et al., 2019] for further details).

Class-driven Criteria. The Class-driven Explanations scenario of Section 2 aims at providing explanations for userselected task functions. Let assume that the user wants the system to learn an explaining function $\psi_{h(i)}$ that is driven by the user-selected $f_{i}$, being $h(\cdot)$ an index mapping function. We propose to enforce the support $X_{h(i)}$ of $\psi_{h(i)}$ to contain $(I F \rightarrow)$ or to be equal to $(I F F \leftrightarrow)$ the space regions in which $f_{i}$ is active. Notice that $f_{i}$ and $\psi_{h(i)}$ have different input domains (perceptual space and concept space, respectively), so we are introducing a constraint between two different representations of the data (see e.g. [Melacci et al., 2009]). Moreover, since the goal of this scenario is to explain $f_{i}$ in terms of the other $f_{u \neq i}$ 's, we mask the $i$-th component of $f(x)$ by setting it to 0 for all $x \in X$. This also avoids trivial solutions in which $\psi_{h(i)}$ only depends on $f_{i}$. We denote by

\footnotetext{
${ }^{1}$ Each penalty term is intended to be weighed by a positive scalar.

${ }^{2}$ We implemented the probability distribution using the softmax operator, scaling the logits with a constant factor to ensure that when $\psi_{j}(x)=1$ all the other $\psi_{z \neq j} 0$ are zero.
}

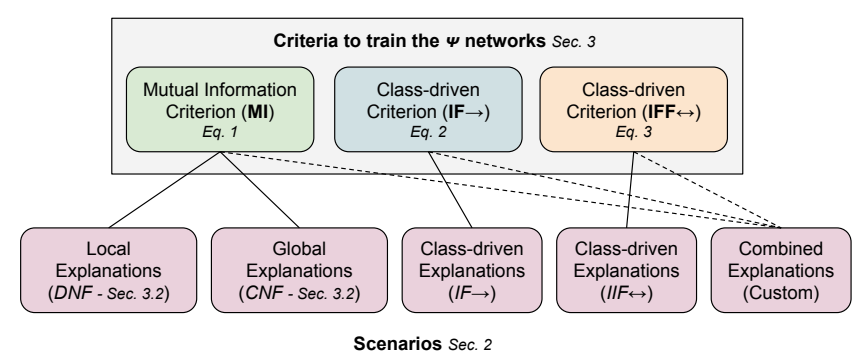

Figure 1: The criteria of the proposed framework and their relations with the use-cases of Section 2.

$P, S \subseteq\{1, \ldots, n\}$ the disjoint sets of task function indexes selected for class-driven $I F \rightarrow$ and $I F F \leftrightarrow$ explanations, respectively. The loss terms that implement the described principles are reported in Eq. 2 and Eq. 3,

$$
\begin{aligned}
& L_{\rightarrow}(\psi, f, X)=\sum_{i \in P, x \in X} \max \left\{0, f_{i}(x)-\psi_{h(i)}(f(x))\right\} \\
& L_{\leftrightarrow}(\psi, f, X)=\sum_{i \in S, x \in X}\left|f_{i}(x)-\psi_{h(i)}(f(x))\right|
\end{aligned}
$$

While Eq. 2 does not penalize those points on which $\psi_{h(i)}(x)>f_{i}(x)$, Eq. 3 specifically enforces the $\psi_{h(i)}$ and $f_{i}$ to be equivalent. In order to avoid trivial solutions of Eq. 2 in which, for instance, $\psi_{h(i)}$ is always 1 , we enforce the superivision loss of $f_{i}$ also on the output of $\psi_{h(i)}$. Notice that these losses never explicitly estimate $X_{h(i)}$.

Class-driven \& Mutual Information-based Criteria. The Combined Explanations scenario of Section 2 is the most general one, and it can be implemented involving all the penalty terms described so far. The MI index can be enforced only on those $\psi_{j}$ 's for which the user is looking for a local explanation, while other explaining functions can be dedicated to class-driven explanations. Interestingly, we can also nest the MI index inside a class-driven explanation, since the user could ask for multiple local explanations for each selected driving class. In this case, multiple $\psi_{j}$ 's are allocated for each driving class, and the MI index is computed assuming the probability distribution of the discrete samples in the concept space to be proportional to the activation of the task function we have to explain. This scenario can be arbitrarily made more complex, and it is out of the scope of this paper to focus on all the possible combinations of the proposed criteria.

Fig. 1 summarizes the role of the described loss terms and their relations with the scenarios of Section 2.

\subsection{First-Order Logic Formulas}

Each explaining function $\psi_{j}$ is a $[0,1]$-valued function defined in $[0,1]^{n}$. At the end of the training stage, each $\psi_{j}$ is converted into a boolean function $\hat{\psi}_{j}$ (this is also considered with a different goal e.g. in [Fu, 1991; Towell and Shavlik, 1993; Tsukimoto, 2000; Sato and Tsukimoto, 2001; Zilke et al., 2016]), and then converted into a FOL formula.

The booleanization step is obtained by approximating any neuron output with its closest integer (assuming sigmoids as activation functions, this value can only be 0 or 1 ) and by repeating this process for each layer, from the output neurons of the task functions up to the output layer of $\psi$. As a result, 


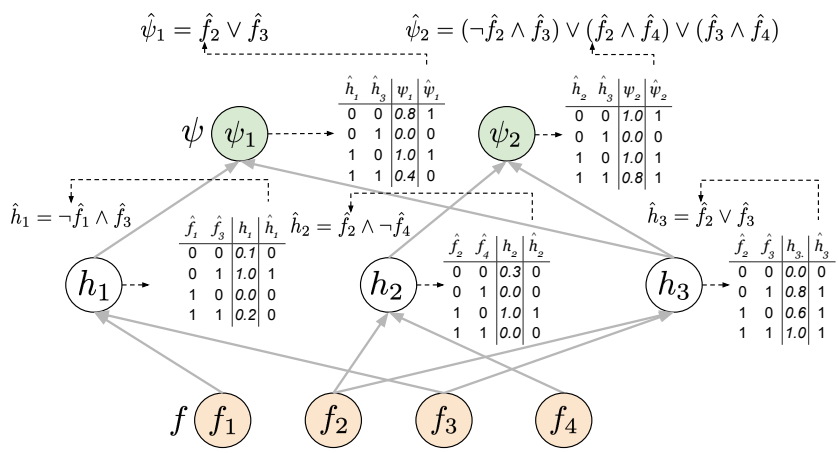

Figure 2: Extracting FOL formulas from each $\psi_{j}$. Hidden and output neurons are paired truth tables (right) and their corresponding logic description (top), as described in Sec. 3.2. The truth tables include the real-value neuron outputs (third column) and their boolean approximation (last column). The FOL descriptions of $\psi_{1}, \psi_{2}$ are the outcome of composing the truth tables of the hidden neurons.

for each neuron we get a boolean function, whose truth-table can be easily rewritten as a boolean formula in Disjunctive Normal Form (DNF), i.e., a disjunction of minterms (conjunction of literals). By composing the formulas attached to each neuron, accordingly to the network structure, we get $\hat{\psi}_{j}$, that is the boolean formula of the output neuron associated to $\psi_{j}$. The whole procedure is illustrated in the example of Figure 2. Clearly, this procedure is efficient only if the fan-in of each neuron is small, a condition that we enforce with the procedure described in Section 3.3.

In the case of Local Explanations (Section 2), each $\psi_{j}$ is close to 1 only in some sup-portions of the space, due to the maximum mutual information criterion, so that the FOL rule $\hat{\psi}_{j}$ will hold true only on $X_{j} \subset X$ (and false otherwise). As a consequence, each explanation is local,

$$
\forall x \in X_{j}, \hat{\psi}_{j}(f(x)), \quad \text { for } j=1, \ldots, m .
$$

The case of Global Explanations (Section 2) is still built on the maximum mutual information criterion. A global explanation (i.e., an explanation holding on the whole input space $X$ ) can be obtained by a disjunction of $\hat{\psi}_{1}, \ldots, \hat{\psi}_{m}$. However, the resulting formula will be generally unclear and quite complex. A possible approach to get a set of global explanations starting from the previous case is then to convert it in Conjunctive Normal Form (CNF), i.e., a conjunction of $K$ disjunctions of literals $\left\{\hat{\psi}_{k}^{\prime}, k=1, \ldots, K\right\}$, $\bigvee_{j=1}^{m} \hat{\psi}_{j}(f(x)) \equiv \bigwedge_{k=1}^{K} \hat{\psi}_{k}^{\prime}(f(x))$. In this case, the following global formulas are valid in all $X$,

$$
\forall x \in X, \hat{\psi}_{k}^{\prime}(f(x)), \quad \text { for } k=1, \ldots, K .
$$

Unfortunately, converting a boolean formula into CNF can lead to an exponential explosion of the formula. However, after having converted each $\hat{\psi}_{j}$ in CNF, the conversion can be computed in polynomial time with respect to the number of minterms in each $\hat{\psi}_{j}$ [Russell and Norvig, 2016].

The Class-driven Explanations (Section 2) naturally generate rules that hold for all $X$ but that are specific for some set of predicates. In particular, Eq. 2 and Eq. 3 enforce $1_{f_{i}} \subseteq X_{h(i)}$ and $1_{f_{i}}=X_{h(i)}$ respectively (for all $i \in P$ and $i \in S$ ), being $1_{f_{i}}$ the characteristic function associated to regions where $f_{i}$ is active. From a logic point of view, we get the validity of the following FOL formulas:

$$
\begin{aligned}
& \forall x \in X, \hat{f}_{i}(x) \rightarrow \hat{\psi}_{h(i)}(x) \quad \text { for } i \in P, \\
& \forall x \in X, \hat{f}_{i}(x) \leftrightarrow \hat{\psi}_{h(i)}(x) \quad \text { for } i \in S,
\end{aligned}
$$

where $\rightarrow$ and $\leftrightarrow$ are the implication and logical equivalence, respectively, and $\hat{f}_{i}$ is the boolean approximation of $f_{i}$.

\subsection{Learning Strategies}

Keeping the fan-in of each neuron in the $\psi$-networks close to small values is a condition that is needed in order to efficiently devise FOL formulas. $L_{1}$-norm-based regularization can be exploited to reduce the number of non-zero-weighed input connections of each neuron. After the training stage, we propose to progressively prune the connections with the smallest absolute values of the associated weights, in order to keep exactly $q \geq 2$ input connections per neuron. This process is performed in an iterative fashion. At each iteration, only one connection per neuron is removed, and a few optimization epochs are performed (using the same loss of the training stage), to let the weights of the $\psi$ functions to readapt after the weight removal. We repeat this process until all the neurons are left with $q$ input connections.

Globally training the whole model involves optimizing the weights of the $f$ - and $\psi$-networks. However, this might lead to low-quality solutions, since the criteria of Section 3.1 might have a dominating role in the optimization. We propose to initially train only the $f$-networks using the available supervisions and the cross-entropy loss, for $E$ epochs. Then, once the selected criteria of Section 3.1 are added to the cost function, both the $f$ and $\psi$-networks are jointly trained. After a first experimentation, we found to be even more efficient to further specialize the latter training, alternating the optimization of the $f$ and $\psi$-networks ( $N_{f}$ epochs for the weights of $f$ and $N_{\psi}$ epochs for the weights of $\psi$, repeated $D$ times).

\section{Experiments}

We considered two different tasks, the joint recognition of objects and objects parts in the PASCAL-Part dataset, and the recognition of face attributes in portrait images of the CelebA data. $^{3}$ In both cases, we compared the quality of the plain classifier (Baseline), against the classifiers augmented with the explanation networks.

Experimental Setup. According to Section 3.3, we set $E=25$, and then 4 learning stages $(D=4)$ are performed, each of them composed of $N_{f}=25$ epochs for the $f$-network (stage $>1$ ) and $N_{\psi}=10$ epochs for the $\psi$-network. For a fair comparison, the baseline classifier is trained for 100 epochs. Each dataset was divided into training, validation, test sets, and we report the (macro) F1 scores measured on the test data. All the main hyperparameters (weights of terms composing the learning criteria of Section 3.1, initial learning rate (Adam optimizer, mini-batch-based stochastic gradient), contribute of the weight decay) have been chosen through a

\footnotetext{
${ }^{3}$ PASCAL-Part: https://www.cs.stanford.edu/ roozbeh/pascal-parts/pascal-parts.html CelebA: http://mmlab.ie.cuhk.edu.hk/projects/CelebA.html
} 


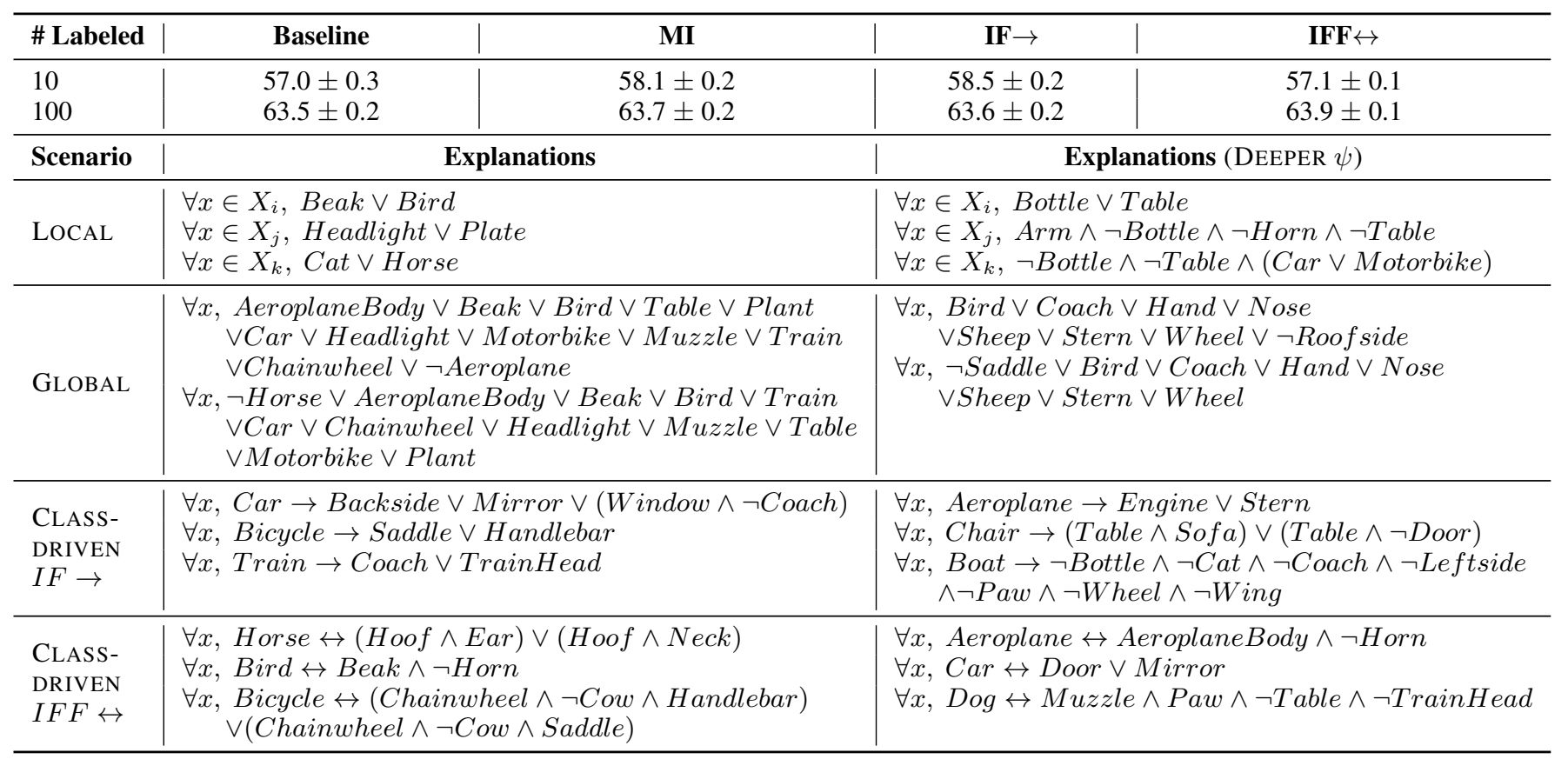

Table 1: PASCAL-Part dataset. Top: macro F1 scores $\%$ ( \pm standard deviation), different learning settings and number of labeled points per-class. Bottom: explanations yielded in different scenarios (two types of $\psi$-network). Functions $\hat{f}_{i}$ 's are indicated with their class-names.

grid search procedure, with values ranging in $\left[10^{-1}, 10^{-4}\right]$, selecting the model that returned the best accuracy on a heldout validation set. Results are averaged over 5 different runs. Each neuron is forced to keep only $q=2$ input connections in the $\psi$-network. Deeper $\psi$-networks are capable of providing more complex explanations, since the compositional structure of the network can relate multiple predicates. We considered two types of $\psi$-networks, with one or two hidden layers (10 units each), respectively, with the exception of the case of $M I$ in which we considered no-hidden layers or one hidden layer (10 units). This is due to the unsupervised nature of the $M I$ criterion, that, when implemented in deeper networks might capture more complex regularities. When class-driven criteria are exploited, we considered an independent neural network to implement each $\psi_{j}$ associated to a driving class. The input space of each of them is different, due to the masking of the driving task function, as described in Section 3.1. When considering the $M I$ criterion only, we used a single $\psi$ network with a number of output units $m$ (one for each $\psi_{j}$ ) ranging from 10 to 50 (cross-validated).

PASCAL-Part. This dataset is composed of 10,103 labeled images of objects (Man, Dog, Car, Train, etc.) and objectparts (Head, Paw, Beak, etc.). We divided them into three splits, composed of 9,092 training images, 505 validation images, 506 test images, respectively (keeping the original class distribution). Following the approach of [Donadello et al., 2017], very specific parts were merged into unique labels, leading to $c=64$ classes, out of which 16 are main objects that contain object-parts from the other classes. From each image, we extracted 2048 features using a ResNet50 backbone network pretrained on ImageNet. We used 100 hidden units and $c$ output units to implement the $f$-network. We tested two different semi-supervised settings in which 10
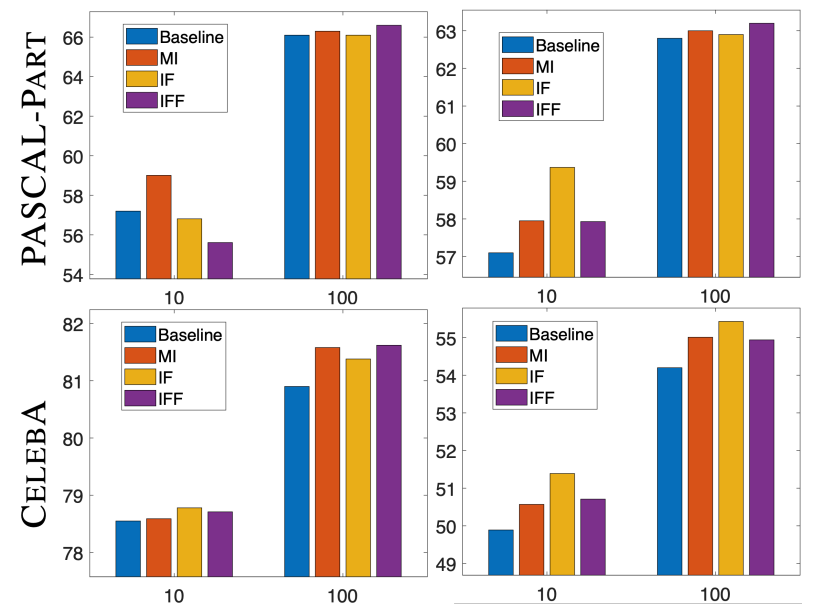

Figure 3: F1 score \% on (a) the driving classes and (b) on the other classes, in function of the number of labeled examples per class.

and 100 labeled examples per-class are respectively provided. The remaining portion of the training data is left unlabeled (it is exploited by the learning criteria of Section 3.1). In the class-driven cases, we considered the main objects as driving classes, so $m=16$. Results are reported in Table 1 , in which the F1 scores (upper portion) and a sample of the extracted rules (lower portion) are shown. The proposed learning criteria lead to an improvement of the classifier performance that is more evident when less supervisions are provided, as expected. We further explored this result, distinguishing between the F1 measured (a) on the driving classes, that are more represented, and (b) on the other classes. Fig. 3 (top) shows that evident improvements (w.r.t. the baseline) can sometimes be due to only one of the two groups of classes, and there is not a clear trend among the criteria. Notice that $\mathrm{s}$ 


\begin{tabular}{|c|c|c|c|c|}
\hline \# Labeled & Baseline & MI & $\mathbf{I F} \rightarrow$ & IFF $\leftrightarrow$ \\
\hline $\begin{array}{l}25 \\
100 \\
\end{array}$ & $\begin{array}{l}54.7 \pm 0.4 \\
60.0 \pm 0.1\end{array}$ & $\begin{array}{l}55.0 \pm 0.3 \\
60.4 \pm 0.2\end{array}$ & $\begin{array}{l}56.1 \pm 0.1 \\
60.9 \pm 0.2\end{array}$ & $\begin{array}{l}55.1 \pm 0.2 \\
60.5 \pm 0.2\end{array}$ \\
\hline Scenario & \multicolumn{2}{|c|}{ Explanations } & \multicolumn{2}{|c|}{ Explanations (DEEPER $\psi$ ) } \\
\hline LOCAL & \multicolumn{2}{|c|}{$\begin{array}{l}\forall x \in X_{i}, \text { Bangs } \wedge \neg \text { Bald } \\
\forall x \in X_{j}, \text { StraightHair } \wedge \text { BushyEyebrows } \\
\forall x \in X_{k}, \text { Female } \wedge \text { Attractive }\end{array}$} & \multicolumn{2}{|c|}{$\begin{array}{l}\forall x \in X_{i}, \text { BlackHair } \wedge \text { Attractive } \wedge \text { Young } \\
\forall x \in X_{j},(\text { Old } \wedge \text { GrayHair }) \vee(\text { Old } \wedge \neg \text { Young }) \\
\forall x \in X_{k}, \text { NoBeard } \wedge \text { Female } \wedge \neg \text { WearNecktie }\end{array}$} \\
\hline GLOBAL & \multicolumn{2}{|c|}{$\begin{array}{l}\forall x, \text { Bangs } \vee \text { BlondHair } \vee \text { Blurry } \vee \text { Goatee } \\
\vee \text { StraightHair } \vee \text { WearHat } \\
\vee \neg \text { Attractive } \vee \neg \text { Female } \vee \neg \text { Male } \\
\forall x, \text { Blurry } \vee \text { Goatee } \vee \text { WearHat } \\
\vee \neg \text { Attractive } \vee \neg \text { BlackHair } \vee \neg \text { BlondHair } \\
\vee \neg \text { Female } \vee \neg \text { StraightHair }\end{array}$} & \multicolumn{2}{|c|}{$\begin{array}{l}\forall x, \text { Beard } \vee \text { BlackHair } \vee \text { Brown Hair } \vee \text { Goatee } \\
\vee \text { HeavyMakeup } \vee \text { Mustache } \vee \text { Old } \vee \\
\text { WearNecktie } \vee \neg \text { Beard } \vee \neg \text { Young } \\
\forall x, \text { HeavyMakeup } \vee \text { Mustache } \vee \text { WearNecktie } \\
\quad \vee \text { Young } \vee \neg \text { Beard } \vee \neg \text { WearLipstick }\end{array}$} \\
\hline $\begin{array}{l}\text { CLASS- } \\
\text { DRIVEN } \\
I F \rightarrow\end{array}$ & \multicolumn{2}{|c|}{$\begin{array}{l}\forall x, \text { Attractive } \rightarrow \text { PaleSkin } \vee \text { RosyCheeks } \\
\vee(\neg \text { Blurry } \wedge \neg \text { Chubby }) \\
\forall x, \text { Beard } \rightarrow \text { Goatee } \vee \text { Sideburns } \\
\forall x, \text { Old } \rightarrow \text { GrayHair } \vee \neg \text { Attractive }\end{array}$} & \multicolumn{2}{|c|}{$\begin{array}{l}\forall x, \text { Male } \rightarrow \text { Beard } \vee \text { FiveOClockShadow } \\
\vee \text { DoubleChin } \vee \neg \text { WearLipstick } \\
\forall x, \text { Bald } \rightarrow \text { RecedingHairline } \wedge \neg \text { Bangs } \\
\wedge \neg \text { RosyCheeks } \wedge \neg \text { WavyHair } \\
\forall x, \text { Female } \rightarrow \text { HeavyMakeup } \vee \text { WearLipstick } \\
\vee(\neg \text { DoubleChin } \wedge \neg \text { WearNecktie })\end{array}$} \\
\hline $\begin{array}{l}\text { CLASS- } \\
\text { DRIVEN } \\
I F F \leftrightarrow\end{array}$ & \multicolumn{2}{|c|}{$\begin{array}{l}\forall x, \text { Bald } \leftrightarrow \neg \text { BlackHair } \wedge \neg \text { BrownHair } \\
\quad \wedge \neg \text { StraightHair } \wedge \neg \text { WavyHair } \\
\forall x, \text { NotBald } \leftrightarrow \text { Bangs } \vee \text { BrownHair } \vee \text { WavyHair } \\
\forall x, \text { Male } \leftrightarrow \neg \text { WearLipstick } \wedge \neg \text { WearNecklace }\end{array}$} & \multicolumn{2}{|c|}{$\begin{array}{l}\forall x, \text { Beard } \leftrightarrow(\text { Goatee } \wedge \text { Mustache }) \\
\quad \vee(\text { Goatee } \wedge \text { Sideburns }) \\
\forall x, \text { Bald } \leftrightarrow \neg \text { Bangs } \wedge \neg \text { StraightHair } \wedge \neg \text { WavyHairg } \\
\forall x, \text { Young } \leftrightarrow(\neg \text { GrayHair } \wedge \text { BigLips }) \\
\quad \vee(\neg \text { GrayHair } \wedge \neg \text { WearNecklace })\end{array}$} \\
\hline
\end{tabular}

Table 2: CelebA dataset. Top: macro F1 scores \% ( \pm standard deviation), different learning settings and number of labeled points per-class. Bottom: explanations yielded in different scenarios (two types of $\psi$-network). Functions $\hat{f}_{i}$ 's are indicated with their class-names.

class-driven criterion not necessarily leads to better drivingtask-functions, while it can also improve the other functions. This is because some driving classes might also participate in explaining other driving classes. The explanations in Table 1 show that deeper $\psi$ networks usually lead to more complex formulas, as expected. Local Explanations depend on the regions covered by the $X_{j}$, and they sometimes involve semantically related classes, that might be simultaneously active on the same region. Global Explanations show possible coverings of the whole classifier output space. We only show 2 sample $\hat{\psi}_{j}^{\prime}$ 's from Eq. 4. They might be harder to follow, since they merge multiple local explanations. In the deeper case we get more compact terms, that, however, are more numerous, i.e., larger $K$. Class-driven Explanations $I F \rightarrow$ and $I F F \leftrightarrow$ provide a semantically coherent description of objects and their parts. Interestingly, these rules usually implement reasonable expectations on this task, with a few exceptions. The $I F F \leftrightarrow$ case is more restrictive than $I F \rightarrow$ (compare Car, Bicycle in the two cases).

CelebA. This dataset is composed of over 200k images of celebrity faces, out of which $45 \%$ are used as training data, $5 \%$ as validation data and $\approx 100 \mathrm{k}$ are used for testing. The dataset is composed of 40 annotated attributes (classes) per image (BlondHair, Sideburns, GrayHair, WavyHair, etc.), that we extended by adding the attributes NotAttractive, NotBald, Female, Beard, Old, as opposite of the already existing Attractive, Bald, Male, NoBeard, Young. In the class-driven criteria, these two sets of attributes are the ones we require to explain $(c=10)$. We exploit the same pre-processing and neural architectures of the previous experiment, evaluating semi-supervised settings with 25 and 100 labeled examples per class. Results are reported in Table 2 and Fig. 3 (bottom). We obtained a slightly less evident improvement of the performance with respect to the baseline, especially in the less-supervised case. This is mostly due to the fact that some classes are associated to high-level attributes (such as Attractive) that might be not easy to generalize from a few supervisions. When distinguishing among the results on driving and not-driving classes (Fig. 3), improvements are more evident. From the Local Explanations in the lower portion of Table 2, we can appreciate that some rules are able to capture in a fully unsupervised way the relationships between, for example, being Attractive and Young, or being Old and with GrayHair. Global Explanations show more differentiated coverings of the classifier output space. Class-driven Explanations $I F \rightarrow$ and $I F F \leftrightarrow$ yield descriptions that, again, are usually in line with common expectations (see Beard, Bald, Male).

\section{Conclusions}

We presented an approach that yields First-Order Logicbased explanations of a multi-label neural classifier, using another neural network that learns to explain the classifier itself. We plan to follow this innovative research direction considering new use-cases and rule types.

\section{Acknowledgements}

This work was partly supported by the PRIN 2017 project RexLearn, funded by the Italian Ministry of Education, University and Research (grant no. 2017TWNMH2). 


\section{References}

[Betti et al., 2019] Alessandro Betti, Marco Gori, and Stefano Melacci. Cognitive action laws: The case of visual features. IEEE transactions on neural networks and learning systems, 2019.

[Bibal and Frénay, 2016] Adrien Bibal and Benoît Frénay. Interpretability of machine learning models and representations: an introduction. In ESANN, 2016.

[Carvalho et al., 2019] Diogo V Carvalho, Eduardo M Pereira, and Jaime S Cardoso. Machine learning interpretability: A survey on methods and metrics. Electronics, 8(8):832, 2019.

[Donadello et al., 2017] Ivan Donadello, Luciano Serafini, and Artur d'Avila Garcez. Logic tensor networks for semantic image interpretation. arXiv preprint arXiv:1705.08968, 2017.

[Doshi-Velez and Kim, 2017] Finale Doshi-Velez and Been Kim. Towards a rigorous science of interpretable machine learning. arXiv preprint arXiv:1702.08608, 2017.

[Došilović et al., 2018] Filip Karlo Došilović, Mario Brčić, and Nikica Hlupić. Explainable artificial intelligence: A survey. In 201841 st International convention on information and communication technology, electronics and microelectronics (MIPRO), pages 0210-0215. IEEE, 2018.

[Freitas, 2014] Alex A Freitas. Comprehensible classification models: a position paper. ACM SIGKDD explorations newsletter, 15(1):1-10, 2014.

[Fu, 1991] LiMin Fu. Rule learning by searching on adapted nets. In $A A A I$, volume 91, pages 590-595, 1991.

[Guidotti et al., 2018] Riccardo Guidotti, Anna Monreale, Salvatore Ruggieri, Franco Turini, Fosca Giannotti, and Dino Pedreschi. A survey of methods for explaining black box models. ACM computing surveys (CSUR), 51(5):93, 2018.

[Gunning, 2017] David Gunning. Explainable artificial intelligence (xai). Defense Advanced Research Projects Agency (DARPA), nd Web, 2, 2017.

[Huysmans et al., 2011] Johan Huysmans, Karel Dejaeger, Christophe Mues, Jan Vanthienen, and Bart Baesens. An empirical evaluation of the comprehensibility of decision table, tree and rule based predictive models. Decision Support Systems, 51(1):141-154, 2011.

[Liu, 2007] Bing Liu. Web data mining: exploring hyperlinks, contents, and usage data. Springer Science \& Business Media, 2007.

[Melacci and Belkin, 2011] Stefano Melacci and Mikhail Belkin. Laplacian support vector machines trained in the primal. Journal of Machine Learning Research, 12(Mar):1149-1184, 2011.

[Melacci and Gori, 2012] Stefano Melacci and Marco Gori. Unsupervised learning by minimal entropy encoding. IEEE transactions on neural networks and learning systems, 23(12):1849-1861, 2012.
[Melacci et al., 2009] Stefano Melacci, Marco Maggini, and Marco Gori. Semi-supervised learning with constraints for multi-view object recognition. In International Conference on Artificial Neural Networks, pages 653-662. Springer, 2009.

[Molnar, 2019] Christoph Molnar. Interpretable machine learning. Lulu. com, 2019.

[Russell and Norvig, 2016] Stuart J Russell and Peter Norvig. Artificial intelligence: a modern approach. Malaysia; Pearson Education Limited,, 2016.

[Sato and Tsukimoto, 2001] Makoto Sato and Hiroshi Tsukimoto. Rule extraction from neural networks via decision tree induction. In IJCNN'01. International Joint Conference on Neural Networks. Proceedings (Cat. No. 01CH37222), volume 3, pages 1870-1875. IEEE, 2001.

[Teso and Kersting, 2019] Stefano Teso and Kristian Kersting. Explanatory interactive machine learning. In Proceedings of the 2019 AAAI/ACM Conference on AI, Ethics, and Society, pages 239-245, 2019.

[Towell and Shavlik, 1993] Geoffrey G Towell and Jude W Shavlik. Extracting refined rules from knowledge-based neural networks. Machine learning, 13(1):71-101, 1993.

[Tsukimoto, 2000] Hiroshi Tsukimoto. Extracting rules from trained neural networks. IEEE Transactions on Neural networks, 11(2):377-389, 2000.

[Witten and Frank, 2005] Ian H Witten and Eibe Frank. Data mining: Practical machine learning tools and techniques 2nd edition. Morgan Kaufmann, San Francisco, 2005.

[Zilke et al., 2016] Jan Ruben Zilke, Eneldo Loza Mencía, and Frederik Janssen. Deepred-rule extraction from deep neural networks. In International Conference on Discovery Science, pages 457-473. Springer, 2016. 